\title{
Total Clearance Observed Normalized by Weight
}

National Cancer Institute

\section{Source}

National Cancer Institute. Total Clearance Observed Normalized by Weight. NCI

Thesaurus. Code 192406.

The observed total body clearance for intravascular administration divided by the weight. 